\title{
Vector Orientation Positioning Method about Tower Spatial Model of Transmission Towers
}

\author{
WANG Fei ${ }^{\mathrm{a}}$, WANG Chang-Zhi ${ }^{\mathrm{b}}$, Liu Hai-Feng ${ }^{\mathrm{c}}$, LIU Ya-Duo ${ }^{\mathrm{d}}$, HU \\ Xiao-Guang ${ }^{\mathrm{e}}$
}

\author{
China Electric Power Research Institute, Xuanwu District, Beijing 100055, China \\ a499422978@qq.com, bwangchangzhi@epri.sgcc.com.cn, cliuhaifeng@epri.sgcc.com.cn, \\ eliuyaduo@epri.sgcc.com.cn, ddkyhxg@163.com
}

\begin{abstract}
Keywords: Transmission tower; Positioning element of tower; Vector orientation method; Cross-product

Abstract: Because of a large number of tower elements in transmission tower, and direction of cross section is complex, it is difficult to quickly set up a space model at the same direction with the actual transmission line tower structure. In this paper, using the method of vector orientation. Define tower element cross section in the local coordinate system, and by targeting vector method locating element of tower in the global coordinate system. The orientation of vector method of tower material has simple steps, easy to implement, and modelling fast.
\end{abstract}

\section{Foreword}

Because of a large number of the tower element in the power transmission tower, section of the tower element consists of the angle steel and the steel tube etc several types, and direction of the cross section of the tower element is very complex, it is difficult to quickly set up a space model which is consistent with direction of the tower element in the actual power transmission tower structure $^{[1]}$, orientation of the tower element in the power transmission tower becomes the main technical bottle neck for modelling of the spatial model of the power transmission tower.

The beam unit modelling of the power transmission tower is realized if orientation of the tower element in the power transmission tower structure is solved. Finite element model of the power transmission tower generally applies the rod unit model for modelling. The rod unit model is characterized with bearing drawing and compressing and not bearing bending moment. This characteristic isn't same as the real model tower, the real model tower is also affected by the bending moment. Therefore orientation direction of the section shall be considered during modelling of the beam unit. Only when orientation of the tower element is basically consistent with actual conditions, accurate simulation effect can be obtained ${ }^{[2]}$.

\section{Key technology}

\subsection{Introduction about technology}

In order to solve orientation issue of the tower element in the power transmission tower structure, this paper invents a orientation method for the tower element in the power transmission tower spatial model. The coordinates of all nodes are firstly defined in the overall coordinate system, and then set up the tower element according to connection relationship of the nodes, placement of the tower element in the space is finally considered, and realize orientation of the power transmission tower element in space.

The general power transmission tower uses the rod unit to set up the model, and placement issue of the tower element in the space isn't considered. But influence of bending moment in the power transmission tower isn't considered when the rod unit is applied to set up the model. In order to consider influence of bending moment received by the power transmission tower, the beam unit is taken advantage to set up the model, and displacement direction of the tower element is considered.

In order to solve orientation of the tower element in the power transmission tower structure and realize quick modelling of the spatial model in the power transmission tower, this invention uses the 
vector positioning method to determine orientation of the tower element in the power transmission tower $^{[3]}$, and realize transfer of the section of the tower element from local coordinate system to overall coordinate system.

The power transmission tower structure consists of the steel tube tower and the angle steel tower according to different tower elements. The section of the steel tube is a central symmetrical section, and it is also axial symmetrical figure. After the section of the steel tube rotates any angle in the plane, its shape is same as that of the original section. Section of the angle steel is a single axle symmetrical figure. After the section of the angle steel rotates a certain angle in the plane, its shape isn't same as that of the original section.

After two end points of the power transmission tower elements are determined in the space, orientation of the angle steel can't be determined uniquely because the section of the angle steel is a single axle symmetrical section, there are many possibility for displacement in the space and it is very complex.

\subsection{Method flow chart}

Flow chart for positioning of the spatial model tower element in the power transmission tower is shown as figure 1. It mainly consists of the following steps: firstly, set up the tower element section of the power transmission tower in the local coordinate system; secondly, select the positioning vector according to actual placement direction of the tower member in the power transmission tower; finally, position the tower element of the power transmission tower in the overall coordinate system according to section of the tower element, positioning vector and the vector positioning method.

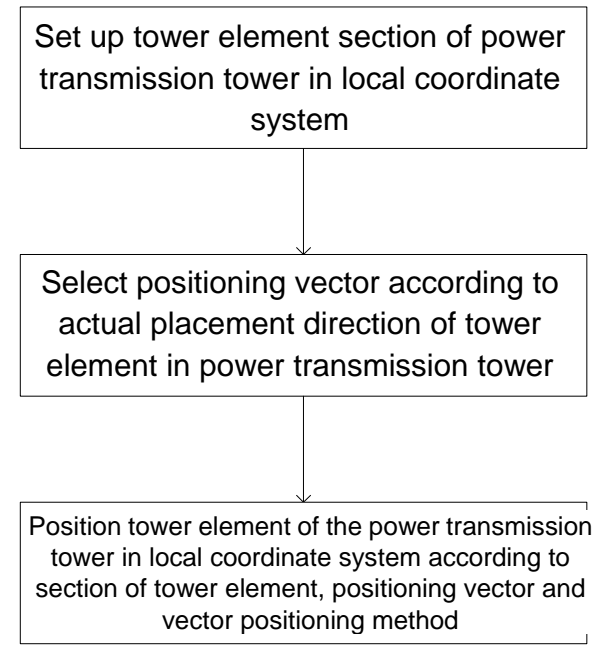

Figure 1 Positioning flow chart of tower element in power transmission tower spatial model

\subsection{Set up overall coordinate system and local coordinate system}

Both the overall coordinate system and the local coordinate system are spatial rectangular coordinate system. The spatial rectangular coordinate system takes one point 0 in the space as origin point, set up three number axis which are vertical two by two: $\mathrm{x}$ axis, $\mathrm{y}$ axis and $\mathrm{z}$ axis. Now the spatial rectangular coordinate system $0 x y z$ is set up, in which point 0 is called as the coordinate origin point, three axis are generally called as coordinate axle, and the plane determined by the coordinate axis is called as the coordinate plane.

The overall coordinate system takes $\mathrm{Z}$ axis as vertical direction, $+\mathrm{Z}$ upward vertically, the $\mathrm{X}-\mathrm{Y}$ plane is horizontal plane, coordinate $(0,0,0)$ point is origin point of the coordinate. The local coordinate system is a spatial rectangular coordinate system which takes direction of the rod member as $\mathrm{x}$ axis, one end point of the rod member as the origin point of the coordinate.

\subsection{Define coordinate of node in power transmission tower}

Shown as figure 2, set up the power transmission tower model in the overall coordinate system, coordinates of the nodes of all rod elements shall be determined in the overall coordinate system, position information of all nodes shall be uniquely determined in the space ${ }^{[4]}$. 
After coordinates of all nodes are defined, the nodes are connected as the rod element according to connection relationship of the power transmission tower. Now two ends of the rod member are determined, and placement of the tower element section in the space isn't uniquely determined, shown as figure 3.

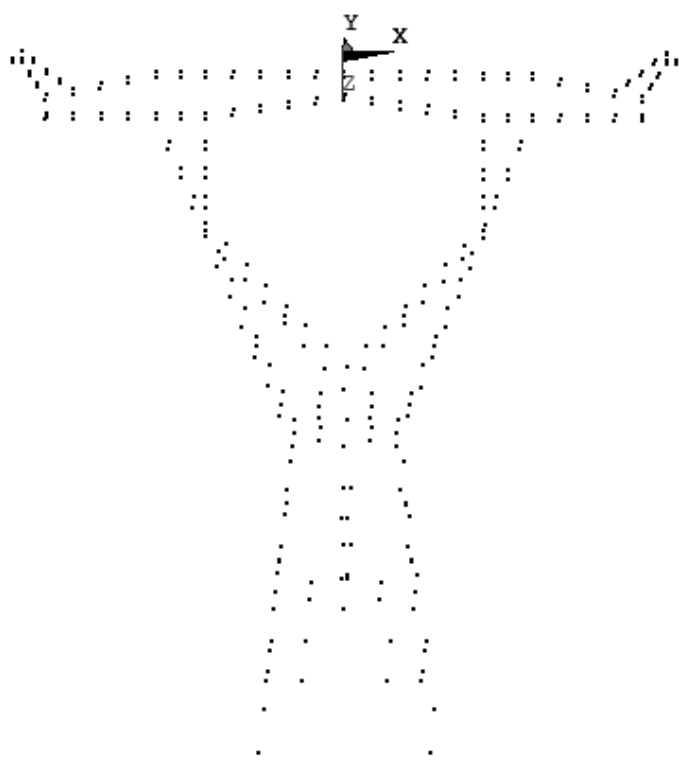

Figure 2 Node of power transmission tower model in overall coordinate system

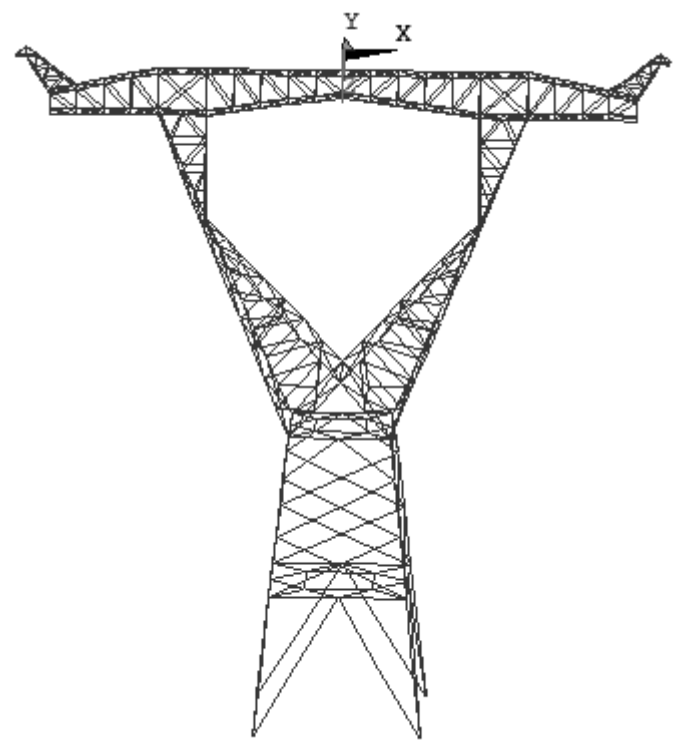

Figure 3 Connection relationship of power transmission tower

\subsection{Set up section of tower element in local coordinate system}

Take the angle steel tower element as a sample, shown as figure 4, section of the tower element is defined in the y-z plane of the local coordinate system, take formal main axis of the tower element section as y axis and $\mathrm{z}$ axis respectively, and set up the tower element section in local coordinate system $^{[5]}$. 


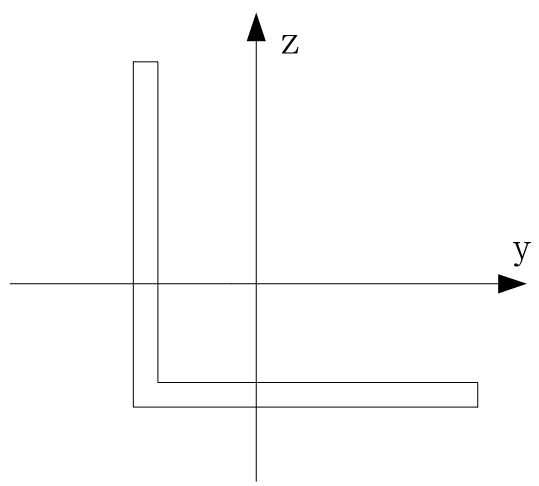

Figure 4 Section of tower element in local coordinate system

\subsection{Position tower element by vector positioning method}

Cross product of two vectors is a vector, and cross product of two vectors is vertical to these two vectors. Shown as figure $5, \mathrm{VxU}$ is a vector, which is vertical to vector $\mathrm{V}$ and vector $\mathrm{U}$.

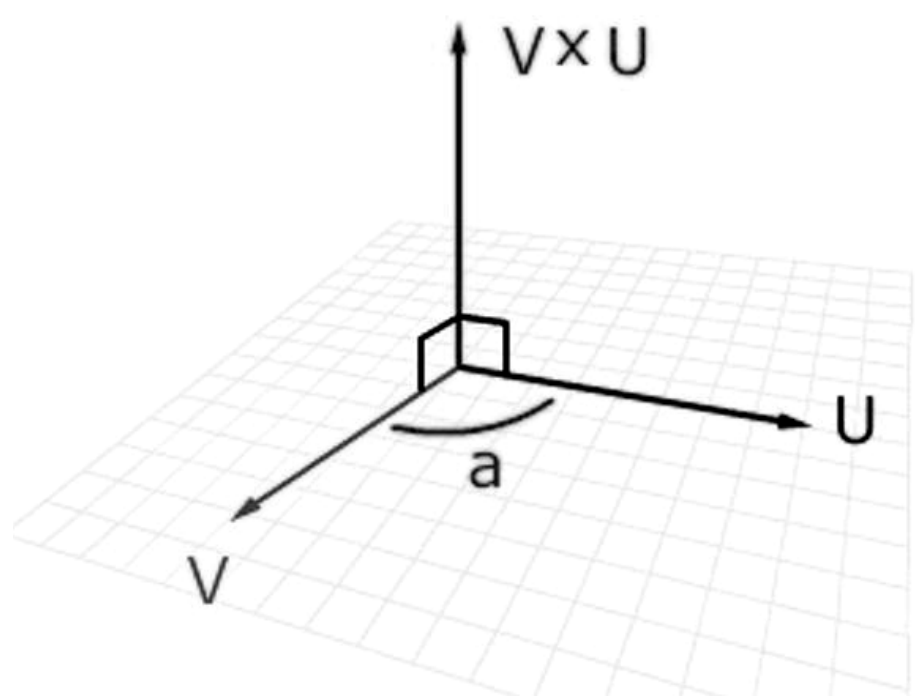

Figure 5 Cross product schematic figure of vector $\mathrm{V}$ and vector $\mathrm{U}$

After section of the power transmission tower element is defined in the local coordinate system, relationship between the local coordinate system and the overall coordinate system shall be determined by the positioning vector, and placement position of the tower element in the space shall be determined.

Shown as figure 6, the local coordinate system is xyz and the overall coordinate system is XYZ. In the local coordinate system, centroid main axles of the tower element are $\mathrm{y}$ axis and $\mathrm{z}$ axis respectively, two end points of the tower element are $\mathrm{i}$ node and $\mathrm{j}$ node, direction from $\mathrm{i}$ node to $\mathrm{j}$ node is direction of the $\mathrm{x}$ axis in the local coordinate system. The section of the $\mathrm{x}$ axis direction and the $\mathrm{z}$ axis direction in the local coordinate system is $\mathrm{xz}$ section, any one vector in the $\mathrm{xz}$ section which isn't in parallel to the $\mathrm{x}$ axle is taken as the positioning vector vecxz, vector value of the positioning vector vecxz in the overall coordinate system is (vecxzX, vecxzY, vecxzZ).

After the positioning vector vecxz is defined, the tower element can be positioned in the overall coordinate system. Connection vector of the tower element from i node to $\mathrm{j}$ node is vector vecx in the overall coordinate system, centroid of main axles $y$ of the tower element is vector vecy in the overall coordinate system, it is obtained through cross product of the spatial positioning vecxz and vector vecx, vector vecz of centroid of main axles $\mathrm{z}$ of the tower element is obtained through cross product of the vector vecy and the vector vecx in the overall coordinate system. 


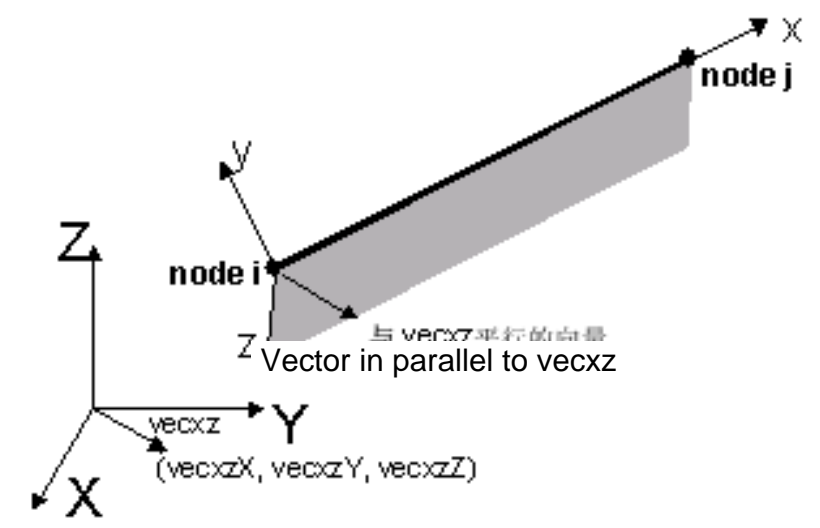

Figure 6 Transfer between local coordinate system and overall coordinate system

\subsection{Selection of positioning vector}

The positioning vector shall be selected according to actual placement of the power transmission tower element. Because the positioning vector can select any one vector in the xz section which isn't in parallel to direction of the $\mathrm{x}$ axis, it can use a few vector positioning tower element. For example: take advantage of vector vecxz $1=(0,0,1)$ to determine the rod element which isn't placed vertically, take advantage of vecxz $2=(1,0,0)$ to determine the rod member which is placed vertically. The spatial model of the power transmission tower is finally formed, shown as figure 7.

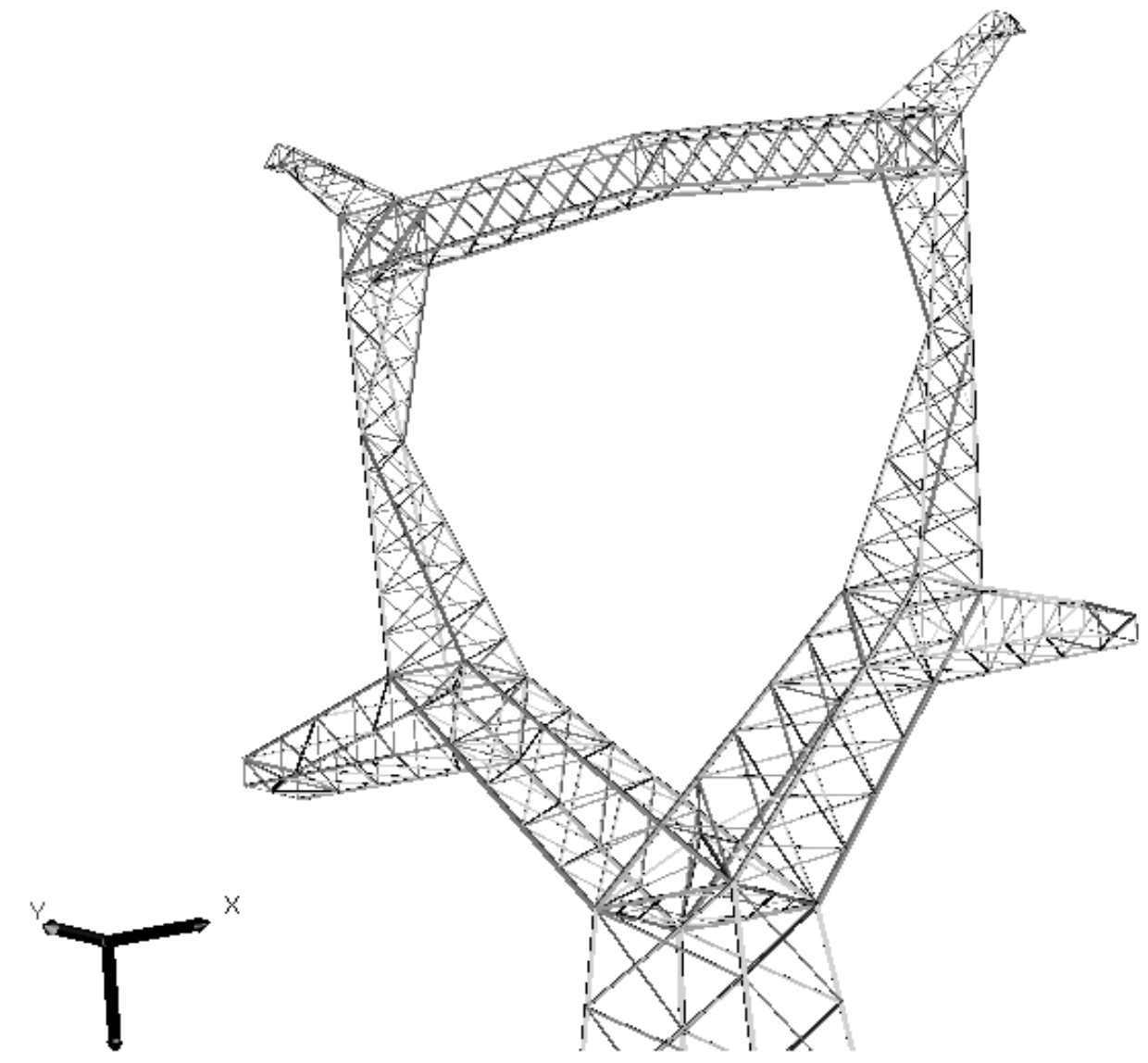

Figure 7 Spatial model of power transmission tower

\section{Advantage of vector positioning method}

Take advantage of the vector positioning method to position the tower element in the spatial model of the power transmission tower and take advantage of positioning vector to position placement 
direction of the tower element, the following advantages are obtained:

1. Step is simple, easy realization;

2. A few vector positioning power transmission tower elements, realize spatial modelling of the power transmission tower;

3. Modelling efficiency is greatly improved when the spatial model is guaranteed to consistent with direction of the tower element in the actual power transmission tower.

4. Realize quick modelling of the spatial model in the angle steel tower, have certain reference meaning for construction of the power transmission tower structure.

\section{Conclusion}

Number of the power transmission tower elements are more, placement direction of the section of the tower element is very complex, it is difficult to quickly set up the spatial model which is consistent with placement direction of the tower element in the actual power transmission tower structure. This paper takes advantage of the vector positioning method, firstly set up the section of the tower element in the power transmission tower in the local coordinate system, and then select positioning vector according to actual placement direction of the tower element in the power transmission tower, and finally position the tower element of the power transmission tower in the overall coordinate system according to section of the tower element, positioning vector and the vector positioning method. The vector positioning method for positioning of the tower element is characterized with simple step, easy realization, quick modelling and less vector positioning power transmission tower element etc advantages.

\section{References}

[1] Li Qinghua, Yang Jingbo. Quick modelling study on power characteristic of ultra-high voltage power transmission tower[J]. Power Construction, 2010, 27(5): 5-0.

[2] Yang Jingbo, Han Junke, Li Maohua etc Selection of calculation model of steel tube tower in ultra-high voltage power transmission line[J]. Grid technology, 2010, 34(1): 1-5.

[3] Zhao Chaoxie, Zhao Dongsong. Structure matrix analysis based on unit positioning vector technology

[4] Lu Mengxiang. Computer aid design of self-support type steel tower[D]. Wuhan: Master thesis of Wuhan University, 2004.

[5]Cook R D,Malkus D S,Plesha M E,et al.Concepts and Applications of Finite Element Analysis. 2002 